\title{
Rationalization of the parameters of the cylindrical bridge support (theoretical basis)
}

\author{
Valery Shmukler ${ }^{*}$, Olena Petrova ${ }^{1}$, and Hammoud Mohammad ${ }^{1}$ \\ ${ }^{1}$ O.M.Beketov National University of Urban Economy in Kharkiv, 17 Marshala Bazhanova Str., \\ Kharkiv, 61002, Ukraine
}

\begin{abstract}
Paper deals with a new constructive solution of a massive concrete pillar intended, mostly, for construction of railway and road bridge supports and with procedure of rationalization of its parameters. Statement and solution of the problem of strength and stability of a concrete support, which has an external mesh cage, reinforcing rings (frames) and an internal cavity is considered. The contact stresses between the concrete core and the metal cage are determined, which, in turn, depend on the attributes of the cage and the frames. The rationalization criterion based on minimizing the potential energy of deformation is introduced. For the indicated task, the variables are the thickness of the mesh cage, the size of its cell, the step and the cross-section of the frames. Support with rational parameters which depend on characteristics of cage and frames gives the lowest material consumption and the highest bearing capacity that is the aim of the approach of investigation.
\end{abstract}

\section{Introduction}

Developing the idea of a concrete support with external reinforcement, presented in [1], this paper proposes the detail approach to the rationalization of its parameters. The effectiveness of external reinforcement made of expanded metal sheet can be extremely increased in case of using the additional transverse steel rings. One can see the interest in the task of finding the rational dependency between parameters of the mesh cage and reinforcing rings. However, together with mentioned above, the task of this work is to establish the bond between the value of the potential energy of deformations (PED) and characteristics of external reinforcement (the cage and the frames at one time) and, using the energy approach [2], to find out the decision which gives minimum value of PED.

\section{Problem Statement}

The problem of rationalization of the structure of a hollow cylindrical, reinforced concrete pillar is considered. Structurally, this pillar represents a concrete element of a tubular type, enclosed in a mesh steel cage. The thickness of the cylinder is such that local buckling is

\footnotetext{
* Corresponding author: petrova.bcd@gmail.com
} 
excluded. In turn, mesh cage is supported by transverse steel rings (frames), set with $a_{c}$ step (Fig. 1).

For high massive supports, the cross-sectional area $A(z)$ should be formulated in accordance with the expression [3]:

$$
A(z)=\frac{\pi}{4}\left(D^{2}-d_{0}^{2} \exp \frac{2 \gamma_{c}}{f_{c}} \cdot z\right)
$$

where $d_{0}$ - diameter of the internal cavity at the origin of the coordinates of the cylindrical system (Fig. 2);

$D$ - external diameter of the support;

$\gamma_{c}$ - specific weight of concrete;

$f_{c}$ - compression tensile strength of concrete.

To the ends of the element (concrete part), axially applied constant stresses of $q$ intensity. Equilibrium of these stresses is equal to $F$.

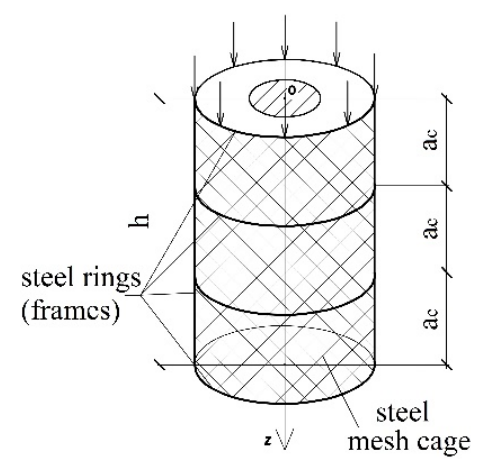

Fig. 1. The support - concrete element in a mesh cage.

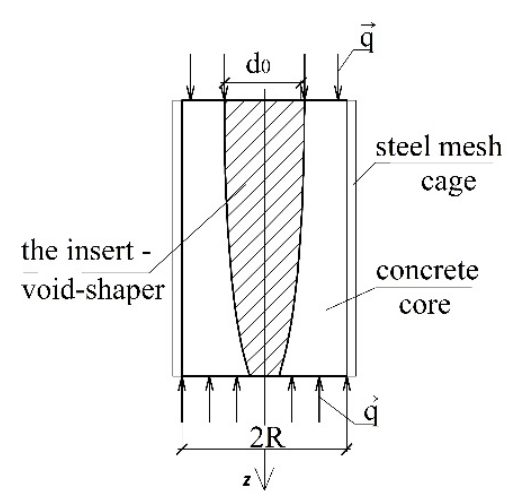

Fig. 2. The longitudinal cross section of the support.

\section{Strength Problem}

Let us denote by $p$ yet unknown contact voltages emerging between the mesh cage and the concrete core. Here it is assumed that radial deformations of the core and circular mesh cage are joint throughout the whole load range (life-cycle).

The transverse cross sections of the considered element (core + cage) under the designated central compression will have the following stress strain state (Fig. 3):

- the core - axially symmetric transverse compression;

- mesh cage - axially symmetric radial tension.

Let's set the following designations:

$R$ - external radius of a concrete core;

$D$ - internal radius of a concrete core;

$r$ - current radius (coordinate of cylindrical system);

$E_{c}, G_{c}$-deformation modules of the 1 st and 2nd kind of concrete core, $G_{c}=\frac{E_{c}}{2\left(1+\mu_{c}\right)}$;

$\mu_{c}-$ Poisson ratio of a concrete core. 

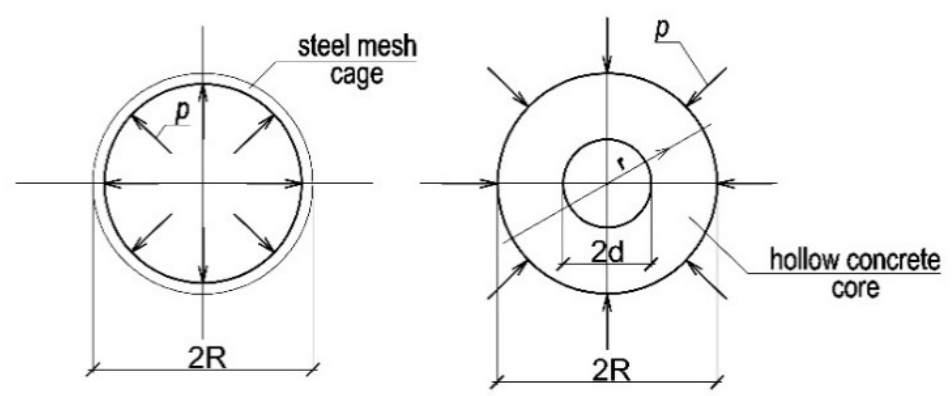

Fig. 3. Cross section of the core and the cage.

\subsection{Concrete core}

To determine the radial deformations of the concrete core, we use the solution of the Lame problem [4].

Radial displacement of the core under compression:

$$
u_{r}=\frac{1}{2 G_{c}\left(R^{2}-d^{2}\right)}\left[\frac{p \cdot d^{2} \cdot R^{2}}{r}+\frac{\left(1-\mu_{c}\right) p R^{2}}{1+\mu_{c}} \cdot r\right]
$$

At the point of contact between the core and the cage $r=R$.

Deformation of the concrete in the transverse direction:

$$
\varepsilon_{r}=\frac{\partial u_{r}}{\partial r}=\frac{p R^{2}}{2 G_{c}\left(R^{2}-d^{2}\right)}\left[d^{2}\left(-\frac{1}{r^{2}}\right)+\frac{1-\mu_{c}}{1+\mu_{c}}\right]
$$

At the point of contact between the core and the cage $r=R$ and

$$
\bar{\varepsilon}_{r}=\frac{(1+\mu) p R^{2}}{\left(R^{2}-d^{2}\right) \cdot E_{c}} \cdot\left(-\frac{d^{2}}{R^{2}}+\frac{(1-\mu)}{(1+\mu)}\right)
$$

Expansion of the core under longitudinal forces in the case of axial symmetry:

$$
\stackrel{\bar{\varepsilon}}{r}_{r}=\frac{1}{2 G_{c}} \cdot\left(-\frac{\mu_{c}}{1+\mu} q\right)=-\frac{\left(1+\mu_{c}\right) \mu}{\left(1+\mu_{c}\right) E_{c}} q
$$

or

$$
{\stackrel{\bar{\varepsilon}}{\varepsilon_{r}}}=\frac{\mu_{c}}{E_{c}}
$$

In case of simultaneous action of $q$ and $p$ (solid cylinder) [5]:

$$
\varepsilon_{r}=\overline{\varepsilon_{r}}+\bar{\varepsilon}_{r}=\frac{p}{E_{c}}-p \mu_{c} \frac{1}{E_{c}}-\frac{\mu_{c} q}{E_{c}}=\frac{p}{E_{c}}-\frac{\mu_{c}}{E_{c}}(p+q)
$$

Considering the direction of compression forces

$$
\varepsilon_{r}=\frac{p}{E_{c}}-\frac{\mu_{c}}{E_{c}}(p+q)
$$


In case of an empty cylinder, after transformations, and considering the results [6]:

$$
\varepsilon_{r}=\frac{\left(1+\mu_{c}\right) R^{2}}{\left(R^{2}-d^{2}\right) E_{c}}\left[\left(\frac{d^{2}}{R^{2}}-\frac{1-\mu_{c}}{1+\mu_{c}}\right) p+\mu_{c} q\right]
$$

For verification when $d=0$ we have:

$$
\varepsilon_{r}=\frac{\left(1+\mu_{c}\right)}{E_{c}}\left[-\frac{1-\mu_{c}}{1+\mu_{c}} p+\mu_{c} q\right]=\frac{1}{E_{c}}\left[-p+\mu_{c} p+\mu_{c} q+\mu_{c}^{2} q\right]
$$

And assuming the validity of $\mu^{2} \ll \varepsilon(\varepsilon>0-$ is a small value), we get

$$
\varepsilon_{r}=\frac{p}{E_{c}}+\frac{\mu_{c}}{E_{c}}(p+q)
$$

which gives a complete coincidence with [5].

\subsection{Steel Mesh Cage}

Let's assume that the size of the cells of mesh cage are small in comparison with radius and height of the support. Then, on the basis of [7], the mesh cage can be roughly replaced by a continuous orthotropic shell. Introducing further a three-orthogonal coordinate system, in the spirit of cited work, we will have:

$$
\begin{gathered}
N_{\alpha}=B_{11} \cdot \varepsilon_{\alpha}+B_{12} \cdot \varepsilon_{\beta}, N_{\beta}=B_{21} \cdot \varepsilon_{\alpha}+B_{22} \cdot \varepsilon_{\beta}, N_{\alpha \beta}=N_{\alpha \beta}=B_{33} \cdot \gamma_{\alpha \beta}, \\
M_{\alpha}=D_{11} \cdot K_{\alpha}+D_{12} \cdot K_{\beta}, \quad M_{\beta}=D_{21} \cdot K_{\alpha}+D_{22} \cdot K_{\beta}, M_{\alpha \beta}=M_{\alpha \beta}=D_{33} \cdot \gamma_{\alpha \beta}, \\
Q_{\alpha}=C_{11} \cdot \Psi_{\alpha}, \quad Q_{\beta}=C_{22} \cdot \Psi_{\beta}
\end{gathered}
$$

where $\varepsilon_{\sigma}, \varepsilon_{\beta}, \varepsilon_{\alpha \beta}$ - deformations of the median surface;

$K_{\alpha}, K_{\beta}, K_{\alpha \beta}$ - bending deformations of the shell (curvature);

$\Psi_{\alpha}, \Psi_{\beta}-$ transverse shear deformation.

$$
\begin{gathered}
B_{11}=2 \frac{B_{h}}{\alpha_{h}} \cdot c^{4}, B_{12}=B_{21}=B_{33}=2 \frac{B_{h}}{\alpha_{h}} \cdot s^{2} \cdot c^{2}, \quad B_{22}=2 \frac{B_{h}}{\alpha_{h}} \cdot s^{4}+\frac{B_{c}}{\alpha_{c}}, \\
D_{m n}=\frac{h^{2}}{12} \cdot B_{m n}(m n=11,12,22,33), C_{11}=2 \frac{C_{h}}{\alpha_{h}} \cdot c^{2}, C_{22}=2 \frac{C_{h}}{\alpha_{h}} \cdot s^{4}+\frac{C_{c}}{\alpha_{c}} \\
B_{h}=\delta_{h} \cdot h \cdot E_{h}, \quad B_{c}=\delta_{c} \cdot h \cdot E_{c}, C_{h}=\delta_{h} \cdot h \cdot G_{h}, C_{c}=\delta_{c} \cdot h \cdot G_{c}
\end{gathered}
$$

In expressions of stiffness (12) for spiral and circular ribs under tension-compression and shear conditions it is indicated (Fig. 4):

$$
s=\sin \varphi, c=\cos \varphi
$$

$\varphi$ is the angle of the inclination of the spiral ribs to the creature;

$h$ - thickness of the mesh structure (height of ribs);

$\delta_{h}, \delta_{c}$ - the width of the spiral and circular ribs, respectively;

$a_{h}, a_{c}$ - the distance between spiral and circular ribs;

$E_{s}, G_{s}$ - elastic and shear modules of ribs (steel);

$n_{h}$ - number of pairs of spiral ribs; 
$D$ is the diameter of the shell.

$$
\alpha=\frac{\pi D}{n_{h}} ; \quad \alpha_{h}=a \cos \varphi ; \quad \alpha_{c}=\frac{a}{2} \operatorname{ctg} \varphi ; \quad l_{h}=\frac{\alpha}{2 \sin \varphi},
$$

where $a$ is the distance between the points of intersection of the symmetrical spiral ribs over the circumference of the cross-section of the shell.

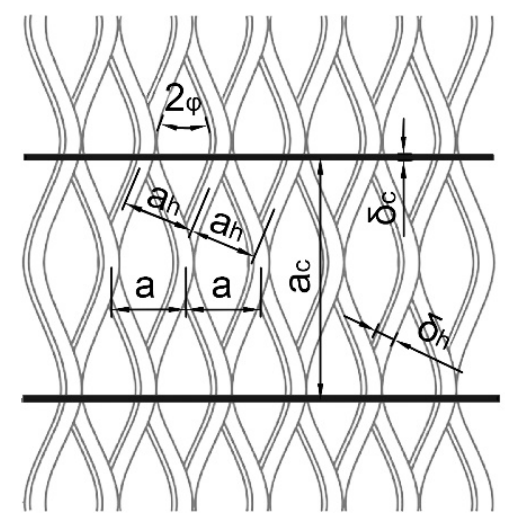

Fig. 4. The main attributes of the cage.

Main attributes of the mesh cage are [8]:

$$
\begin{gathered}
\varepsilon_{\alpha}^{e}=-\frac{1}{B_{e}} B_{12}^{e} N_{\beta} \\
\varepsilon_{\beta}^{e}=\frac{1}{B_{e}} B_{11}^{e} N_{\beta} ; \quad \varepsilon_{\beta}^{e}=\frac{B_{11}}{B_{e}} p R \\
B_{e}=B_{11}^{e} B_{22}^{e}-\left(B_{12}^{e}\right)^{2}=B_{11}^{e} B_{22}^{e}-B_{12}^{e} \cdot B_{21}^{e} \\
\text { and } \quad N_{\beta}=p R, \quad N_{\alpha}=0.0 .
\end{gathered}
$$

Equating the radial deformation of the concrete core to the annular deformation of steel cage, we get:

$$
\frac{\left(1+\mu_{c}\right) \cdot R^{2}}{E_{c} \cdot\left(R^{2}-d^{2}\right)}\left\{\left[\frac{d^{2}}{R^{2}}-\frac{1-\mu_{c}}{1+\mu_{c}}\right] \cdot p+\mu_{c} \cdot q\right\}=\frac{B_{11}^{s}}{B_{e}^{s}} \cdot p R
$$

Let's set the designation:

$$
\gamma_{1}=\frac{\left(1+\mu_{c}\right) \cdot d^{2}}{E_{c} \cdot\left(R^{2}-d^{2}\right)}, \quad \gamma_{2}=\frac{\left(1-\mu_{c}\right) \cdot R^{2}}{E_{c} \cdot\left(R^{2}-d^{2}\right)}, \quad \gamma_{3}=\frac{\mu_{c}\left(1+\mu_{c}\right) \cdot R^{2}}{E_{c} \cdot\left(R^{2}-d^{2}\right)}, \quad \gamma_{4}=\frac{B_{11}^{s}}{B_{e}^{s}} \cdot R
$$

Then

$$
\left(-\gamma_{1}+\gamma_{2}+\gamma_{4}\right) \cdot p=\gamma_{3} \cdot q
$$

Or 


$$
p=\frac{\gamma_{3}}{\left(\gamma_{2}+\gamma_{4}-\gamma_{1}\right)} \cdot q
$$

Futher, considering the relationship between the contact tensions $\mathrm{p}$ and the longitudinal (given) stresses q, written for the isotropic shell, we obtain [5]:

$$
p=\frac{\mu_{c}}{\frac{R}{h_{\text {red }}} \cdot n+1-\mu_{c}} \cdot q
$$

where $n=\frac{E_{c}}{E_{s}}-$ reduction factor;

$h_{\text {red }}$ - reduced thickness of the mesh cage.

Having a sort of cut sheets, it is possible on the basis of (20) to determine the contact tension between the cage and the core, depending on the main attributes of the sheet and the step of the steel rings (frames).

\subsection{Stress State of the Core}

To estimate the stress state of the core we define the main stresses, considering that the components of the stress tensor, in this case, are the following [2, 4]:

$$
\begin{gathered}
\sigma_{r}=\frac{-R^{2}}{R^{2}-d^{2}} \cdot\left(\frac{d^{2}}{r^{2}}-1\right) \cdot p ; \\
\sigma_{\beta}=\frac{-R^{2}}{R^{2}-d^{2}} \cdot\left(\frac{d^{2}}{r^{2}}+1\right) \cdot p ; \\
\sigma_{z}=-q ; \\
\sigma_{r z}=\sigma_{\beta z}=\sigma_{r \beta}=0 .
\end{gathered}
$$

As a consequence, invariants of the stress tensor will be:

$$
\begin{gathered}
I_{1}\left(T_{\sigma}\right)=\sigma_{r}+\sigma_{\beta}+\sigma_{z}=-\frac{2 R^{2}}{R^{2}-d^{2}} \cdot p-q \\
I_{1}\left(T_{\sigma}\right)=-\sigma_{r} \cdot \sigma_{\beta}-\sigma_{r} \cdot \sigma_{z}-\sigma_{\beta} \cdot \sigma_{z}=\frac{-R^{2} \cdot p}{R^{2}-d^{2}}\left(\frac{d^{2}}{r^{2}}-1\right)\left[-\frac{R^{2} \cdot p}{R^{2}-d^{2}}\left(\frac{d^{2}}{r^{2}}+1\right)\right]+ \\
+\frac{R^{2} \cdot p \cdot q}{R^{2}-d^{2}}\left(\frac{d^{2}}{r^{2}}-1\right)-\frac{R^{2} \cdot p \cdot q}{R^{2}-d^{2}}\left(\frac{d^{2}}{r^{2}}+1\right)=\frac{R^{4} \cdot p^{2}}{\left(R^{2}-d^{2}\right)^{2}}\left(\frac{d^{4}}{r^{2}}-1\right)-\frac{2 R^{2} \cdot p \cdot q}{R^{2}-d^{2}}= \\
=\frac{R^{2} \cdot p}{R^{2}-d^{2}}\left[\frac{p\left(d^{4}-r^{4}\right) R^{2}}{\left(R^{2}-d^{2}\right) r^{4}}-2 q\right]
\end{gathered}
$$




$$
I_{3}\left(T_{\sigma}\right)=\sigma_{r} \cdot \sigma_{\beta} \cdot \sigma_{z}=\frac{R^{4}}{\left(R^{2}-d^{2}\right)^{2}}\left(\frac{d^{4}}{r^{4}}-1\right) \cdot p^{2} q
$$

Decisive equation concerning main stresses becomes:

$$
\sigma^{3}-I_{1}\left(T_{\sigma}\right) \cdot \sigma^{2}-I_{2}\left(T_{\sigma}\right) \cdot \sigma-I_{3}\left(T_{\sigma}\right)=0
$$

Or with respect of (26-28):

$$
\sigma^{3}+\left[\frac{2 R^{2}}{R^{2}-d^{2}} \cdot p+q\right] \cdot \sigma^{2}-\frac{R^{2} \cdot p}{R^{2}-d^{2}}\left[\frac{\left(d^{4}-r^{4}\right) \cdot R^{2} p}{\left(R^{2}-d^{2}\right) \cdot r^{4}}-2 q\right] \cdot \sigma-\frac{R^{4}}{\left(R^{2}-d^{2}\right)^{2}}\left(\frac{d^{4}}{r^{4}}-1\right)=0
$$

At the point where $r=d$, (29) is converted to

$$
\sigma^{2}+\left[\frac{2 R^{2}}{R^{2}-d^{2}} \cdot p+q\right] \cdot \sigma+\frac{2 R^{2} \cdot p \cdot q}{R^{2}-d^{2}}
$$

Herefrom

$$
\sigma_{1,2}=-\frac{1}{2}\left(\frac{2 R^{2}}{R^{2}-d^{2}} \cdot p+q\right) \pm \sqrt{\frac{1}{4}\left(\frac{2 R^{2}}{R^{2}-d^{2}} \cdot p+q\right)^{2}-\frac{2 R^{2} \cdot p \cdot q}{R^{2}-d^{2}}}
$$

At the point where $r=R$, the equation (29) will have a look of:

$$
\sigma^{3}+\left[\frac{2 R^{2}}{R^{2}-d^{2}} \cdot p+q\right] \cdot \sigma^{2}+\frac{R^{2} \cdot p}{R^{2}-d^{2}} \cdot\left(\frac{R^{2}+d^{2}}{R^{2}} \cdot p-2 q\right) \sigma+\frac{R^{2}+d^{4}}{R^{2}-d^{2}} \cdot p^{2} q=0
$$

As a result of the solution (29), (30), (32), we obtain the main stresses:

$$
\sigma_{1} \geq \sigma_{2} \geq \sigma_{3}
$$

Knowing the main stresses, one can determine the density of the potential energy of deformations:

$$
e=\frac{1}{2 E_{c}}\left[\sigma_{1}^{2}+\sigma_{2}^{2}+\sigma_{3}^{2}-2 \mu_{c}\left(\sigma_{1} \cdot \sigma_{2}+\sigma_{1} \cdot \sigma_{3}+\sigma_{2} \cdot \sigma_{3}\right)\right],
$$

and, finally, the potential energy (PED)

$$
U=\int_{V} e d V
$$

where $U$ - potential energy of deformation;

$V$ - the volume of the support.

Apparently that due to presented algorithm, the main stresses, the density of the potential energy of deformation and PED also depend on the parameters of the mesh cage.

\subsection{Criterion and procedure of rationalization}

As a criterion for rationalization of the considered design, it is accepted that: 


$$
U \rightarrow \text { inf }
$$

with limitation

$$
e_{i} \leq e_{n c}
$$

where $e_{n c}$ is the normalized value of the density of deformation energy (DPED);

$e_{i}$ - DPED in the $i$-th point of the rack.

$$
e_{n} \leq \gamma \cdot e_{u}
$$

where $e_{u}$ is the limiting value of the density of deformation energy (DPED);

$\gamma$ - statistically substantiated safety ratio, in the first approximation it is acceptable to take $\gamma$ $=0,77$.

In turn, the DPED is given in accordance with the Vasil'kov - Shmukler criterion [9]:

$$
e_{u}=0,5 \chi_{\sigma}^{2}\left[\left(\chi_{\sigma}+1\right) e_{c u}-\left(\chi_{\sigma}-1\right) e_{t u}\right]+\left(1-\chi_{\sigma}^{2}\right) e_{s h u}
$$

where $\chi_{\sigma}=\frac{2 \sigma_{2}-\sigma_{1}-\sigma_{3}}{\sigma_{1}-\sigma_{3}}-$ the Lode-Nadai parameter of the stress state

$e_{s u}, e_{t u}, e_{s h u}$ - partial values of the density of deformation energy for compression, tension, and shear, respectively.

Let's denote that for uniaxial tension:

$\sigma_{1} \neq 0 ; \sigma_{2}=0 ; \sigma_{3}=0 ; \chi_{\sigma}=-1.0$,

for uniaxial compression:

$\sigma_{1}=0 ; \sigma_{2}=0 ; \sigma_{3} \neq 0 ; \chi_{\sigma}=-1.0$,

for uniaxial shear:

$\sigma_{1}=\sigma_{3} ; \sigma_{2}=0 ; \chi_{\sigma}=0.0$.

Each partial value of the limiting density of the deformation's energy is determined by:

$$
e_{i u}=\int_{0}^{\varepsilon_{u}} \sigma(\varepsilon) d \varepsilon, \quad i=c, t, s h
$$

where $\sigma_{i}(\varepsilon)$ is the known dependence between stresses and deformations, which is obtained experimentally for compression (c), tensile (t) and shear (sh);

$\varepsilon_{\mathrm{u}}$ is the limiting deformation.

An important point here is the fact (and (40) expression reflects it) that, in general case, the limiting deformation does not correspond to the maximum tension (due to physical nonlinearity).

And since the connection between stresses and deformations for concrete is nonlinear, the polynomial of the fifth degree is accepted for its description $[9,10]$.

In this case, deformation diagrams of concrete for compression, tension and shear are taken on the basis of:

$$
\sigma_{L}=\alpha_{1} \cdot \varepsilon_{L}+\alpha_{2} \cdot \varepsilon_{L}^{2}+\alpha_{3} \cdot \varepsilon_{L}^{3}+\alpha_{4} \cdot \varepsilon_{L}^{4}+\alpha_{5} \cdot \varepsilon_{L}^{5}
$$

\section{Conclusions}

Listed, in aggregate, predetermines the following conclusion: 
The developed approach of assessment of the effect of a mesh cage reinforced by frames on the stress-strain state of a cylindrical concrete support allows one to determine the rational parameters of the reinforcement elements (characteristics of the mesh sheet and steel frames) on the basis of the energy portrait of a structure based on new energy methods [9].

\section{References}

1. O. Petrova, O.Lugchenko, A. Nazhem. MATEC Web of Conferences 116, 02025 (2017).

2. G.V. Vasilkov. Evolutionary theory of the life cycle of mechanical systems. Theory of constructions. Moscow: LKI (2008).

3. V.S. Shmukler, O.O. Petrova, O.V. Sinkovska. Scientific Bulletin of Construction. Kh.: KhNUBA, KhOTV ABU, 74, 148-156 (2013).

4. V.G. Rekach. Kerivnytstvo do vyrishennia zadach z teorii pruzhnosti. - Moscow: Higher School (1966).

5. B.N. Zhemochkin. Teoria pruzhnosti. - M .: State Publishing House of Literature on Construction and Architecture (1957).

6. Tymoshenko, S.P. Opir materialiv. - Moscow: Nauka Volume I (1965).

7. A.V. Azarov. Composites and nanostructures, Moscow, 7(3), 151-161 (2015).

8. A.V. Egorov, A.V. Azarov. Electronic Magazine of Labor "MAI", 73, 1-16 (2014).

9. V.S. Shmukler. Collection of scientific works of the Ukrainian State University of Railway Transport. - Kh., 167, 54-70 (2017).

10. M. Hammoud, V. Shmukler, O. Petrova. Proc. of the 12th fib International PhD Symposium in Civil Engineering. CTU, Prague, Czech Republic, 593-599 (2018). 